\title{
Determining Patterns in Neural Activity for Reaching Movements Using Nonnegative Matrix Factorization
}

\author{
Sung-Phil Kim \\ Department of Electrical and Computer Engineering, University of Florida, Gainesville, FL 32611, USA
}

Email:phil@cnel.ufl.edu

Yadunandana N. Rao

Motorola Inc., FL, USA

Email:yadu@cnel.ufl.edu

\section{Deniz Erdogmus}

Department of Computer Science and Biomedical Engineering, Oregon Health \& Science University, Beaverton, OR 97006, USA

Email:derdogmus@ieee.org

\section{Justin C. Sanchez}

Department of Pediatrics, Division of Neurology, University of Florida, Gainesville, FL 32611, USA

Email: justin@cnel.ufl.edu

\section{Miguel A. L. Nicolelis}

Department of Neurobiology, Center for Neuroengineering, Duke University, Durham, NC 27710, USA Emails:derdogmus@ieee.org; nicoleli@neuro.duke.edu

\author{
Jose C. Principe \\ Department of Electrical and Computer Engineering, University of Florida, Gainesville, FL 32611, USA \\ Email:principe@cnel.ufl.edu
}

Received 31 January 2004; Revised 23 March 2005

\begin{abstract}
We propose the use of nonnegative matrix factorization (NMF) as a model-independent methodology to analyze neural activity. We demonstrate that, using this technique, it is possible to identify local spatiotemporal patterns of neural activity in the form of sparse basis vectors. In addition, the sparseness of these bases can help infer correlations between cortical firing patterns and behavior. We demonstrate the utility of this approach using neural recordings collected in a brain-machine interface (BMI) setting. The results indicate that, using the NMF analysis, it is possible to improve the performance of BMI models through appropriate pruning of inputs.
\end{abstract}

Keywords and phrases: brain-machine interfaces, nonnegative matrix factorization, spatiotemporal patterns, neural firing activity.

\section{INTRODUCTION}

Brain-machine interfaces (BMIs) are an emerging field that aims at directly transferring the subject's intent of movement to an external machine. Our goal is to engineer devices that are able to interpret neural activity originating in the motor cortex and generate accurate predictions of hand position. In the BMI experimental paradigm, hundreds of microelectrodes are implanted in the premotor, motor, and posterior parietal areas and the corresponding neural activity is recorded synchronously with behavior (hand reaching and grasping movements). Spike detection and sorting algorithms are used to determine the firing times of single neurons. Typically, the spike-time information is summarized into bin counts using short windows (100 milliseconds in this paper). A number of laboratories including our own have demonstrated that linear and nonlinear adaptive system identification approaches using the bin count input 
can lead to BMIs that effectively predict the hand position and grasping force of primates for different movement tasks $[1,2,3,4,5,6,7,8]$. The adaptive methods studied thus far include moving average models, time-delay neural networks (TDNNs), Kalman filter and extensions, recursive multilayer perceptrons (RMLPs), and mixture of linear experts gated by hidden Markov models (HMMs).

BMIs open up an important avenue to study the spatiotemporal organization of spike trains and their relationships with behavior. Recently, our laboratory has investigated the sensitivity of neurons and cortical areas based on their role in the mapping learned by the RMLP and the Wiener filter [7]. We examined how each neuron contributes to the output of the models, and found consistent relationships between cortical regions and segments of the hand trajectory in a reaching movement. This analysis indicated that, during each reaching action, specific neurons from the posterior parietal, the premotor dorsal, and the primary motor regions sequentially became dominant in controlling the output of the models. However, this approach relies on determining a suitable model, because it explicitly uses the learned model to infer the dependencies.

In this paper, we propose a model-independent methodology to study spatiotemporal patterns between neuronal spikes and behavior utilizing nonnegative matrix factorization (NMF) $[9,10]$. In its original applications, NMF was mainly used to provide an alternative method for determining sparse representations of images to improve recognition performance $[10,11]$. d' Avella and Tresch have also proposed an extension of NMF to extract time-varying muscle synergies for the analysis of behavior patterns of a frog [12]. The nonnegativity constraints in NMF result in the unsupervised selection of sparse bases that can be linearly combined (encoded) to reconstruct the original data. Our hypothesis is that NMF can similarly yield sparse bases for analyzing neural firing activity, because of the intrinsic nonnegativity of the bin counts and the sparseness of spike trains.

The application of NMF to extract local features of neural spike counts follows the method of obtaining sparse bases to describe the local features of face images. The basis vectors provided by NMF and their temporal encoding patterns are examined to determine how the activities of specific neurons localize to each segment of the reaching trajectory. We will show that the results from this model-independent analysis of the neuronal activity are consistent with the previous observations from the model-based analysis.

\section{NONNEGATIVE MATRIX FACTORIZATION}

NMF is a procedure to decompose a nonnegative data matrix into the product of two nonnegative matrices: bases and encoding coefficients. The nonnegativity constraint leads to a parts-based representation, since only additive, not subtractive, combinations of the bases are allowed. An $n \times m$ nonnegative data matrix $\mathbf{X}$, where each column is a sample vector, can be approximated by NMF as

$$
\mathbf{X}=\mathbf{W H}+\mathbf{E},
$$

where $\mathbf{E}$ is the error and $\mathbf{W}$ and $\mathbf{H}$ have dimensions $n \times r$ and $r \times m$, respectively. W consists of a set of $r$ basis vectors, while each column of $\mathbf{H}$ contains the encoding coefficients for every basis for the corresponding sample. The number of bases is selected to satisfy $r(n+m)<n m$ so that the number of equations exceed that of the unknowns.

This factorization can be described in terms of columns as

$$
\mathbf{x}_{j} \approx \mathbf{W h}_{j}, \quad \text { for } j=1, \ldots, m,
$$

where $\mathbf{x}_{j}$ is the $j$ th column of $\mathbf{X}$ and $\mathbf{h}_{j}$ is the $j$ th column of $\mathbf{H}$. Thus, each sample vector is a linear combination of basis vectors in $\mathbf{W}$ weighted by $\mathbf{h}_{j}$. The nonnegative constraints on $\mathbf{W}$ and $\mathbf{H}$ allow only additive combination of basis vectors to approximate $\mathbf{x}_{j}$. This constraint allows the visualization of the basis vectors as "part" of the original sample [10]. This is contrary to factorization by PCA, where negative basis vectors are allowed.

The decomposition of $\mathbf{X}$ into $\mathbf{W}$ and $\mathbf{H}$ can be determined by optimizing an error function between the original data matrix and the decomposition. Two possible cost functions used in the literature are the Frobenius norm of the error matrix $\|\mathbf{X}-\mathbf{W H}\|_{F}^{2}$ and the Kullback-Leibler divergence $D_{K L}(\mathbf{X} \| \mathbf{W H})$. The nonnegativity constraint can be satisfied by using multiplicative update rules discussed in [10] to minimize these cost functions. In this paper, we will employ the Frobenius norm measure, for which the multiplicative update rules that converge to a local minimum are given below:

$$
\begin{aligned}
\mathbf{H}_{\mu j}(k+1) & =\mathbf{H}_{\mu_{j}}(k) \frac{\left(\mathbf{W}^{T} \mathbf{X}\right)_{\mu_{j}}}{\left(\mathbf{W}^{T} \mathbf{W}\right)_{\mu_{j}}}, \\
\mathbf{W}_{i \mu}(k+1) & =\mathbf{W}_{i \mu}(k) \frac{\left(\mathbf{X H}^{T}\right)_{i \mu}}{\left(\mathbf{W H H}^{T}\right)_{i \mu}} .
\end{aligned}
$$

$\mathbf{A}_{a b}$ denotes the element of a matrix $\mathbf{A}$ at $a$ th row and $b$ th column. It has been proven in [9] that the Frobenius norm cost function is nonincreasing under this update rule.

\section{FACTORIZATION OF THE NEURONAL ACTIVITY MATRIX}

We will now apply the multiplicative update rule in (2) to the neuronal bin-count matrix (created by real neural recordings of a behaving primate). The goal is to determine nonnegative sparse bases for the neural activity, from which we wish to deduce the local spatial structure of the neural population firing activity. These bases also point out common population firing patterns corresponding to the specific behavior. In addition, the resulting factorization yields a temporal encoding matrix that indicates how the instantaneous neural activity is optimally constructed from these localized representations. Since we are interested in the relationship between the neural activity and behavior, we would like to study the coupling between this temporal encoding pattern with the movement of the primate, as well as the contribution of the specific bases vectors, which represent neural populations. 
TABLE 1: Distribution of neurons and cortical regions.

\begin{tabular}{l|cccc|c}
\hline & \multicolumn{9}{|c|}{ Monkey-1 } & \multicolumn{2}{c}{ Monkey-2 } \\
\hline Regions & PP & M1(area 1) & PMd & M1(area 2) & M1 \\
Neurons & $1 \sim 33$ & $34 \sim 54$ & $55 \sim 81$ & $82 \sim 104$ & $1 \sim 37$ \\
\hline
\end{tabular}

\subsection{Data preparation}

Synchronous, multichannel neuronal spike trains were collected at Duke University using two female owl monkeys (Aotus trivirgatus): Belle (monkey-1) and Carmen (monkey2). ${ }^{1}$ Microwire electrodes were implanted in cortical regions where motor associations are known [1, 13]. During the neural recording process, up to sixty-four electrodes were implanted in posterior parietal (PP)-area 1, primary motor (M1)-area 2, area 4, and premotor dorsal (PMd)-area 3, each receiving sixteen electrodes. From each electrode, one to four neurons can be discriminated. The firing times of individual neurons were determined using spike detection and sorting algorithms [14] and were recorded while the primate performed a $3 \mathrm{D}$ reaching task that consists of a reach to food followed by eating. The primate's hand position was also recorded using multiple fiber optic sensors (with a shared time clock) and digitized with a $200 \mathrm{~Hz}$ sampling rate [1]. These sensors were contained in the plastic strip of which bending and twisting modified the transmission of the light through the sensors in order to record positions in 3D space more accurately. The neuronal firing times were binned in nonoverlapping windows of 100 milliseconds, representing the local firing rate for each neuron. In this recording session of approximately 20 minutes (12000 bins), 104 neurons for monkey- 1 and 54 neurons for monkey- 2 could be discriminated (whose distribution to cortical regions is provided in Table 1 from [13]), and there were 71 reaching actions for monkey-1 and 65 for monkey-2, respectively. These reaching movements consist of three natural segments shown in Figure 1.

Based on the analysis of Wessberg et al. [1], the instantaneous movement is correlated with the current and the past neural data up to 1 second (10 bins). Therefore, for each time instant, we form a bin-count vector by concatenating 10 bins of firing counts (which correspond to 10-tap delay line in a linear filter) from every neuron. Hence, if $x_{j}(i)$ represents the $i$ th bin of neuron $j$, where $i \in\{1, \ldots, 12000\}$, a bin-count vector at time instance $i$ is represented by $\mathbf{x}(i)=$ $\left[x_{1}(i), x_{1}(i-1), \ldots, x_{1}(i-9), x_{2}(i), \ldots, x_{n}(i-9)\right]^{T}$, where $n$ is the number of neurons. Since we are interested in determining repeated spatiotemporal firing patterns during the reaching movements, only the bin counts from time instances where the primate's arm is moving are considered. There is a possibility that in the selected training set some neurons never fire. The rows corresponding to these neurons must be removed from the bin-count matrix, since they tend to cause

\footnotetext{
${ }^{1}$ All experimental procedures conformed to the National Academy Press Guide for the Care and Use of Laboratory Animals and were approved by the Duke University Animal Care and Use Committee.
}

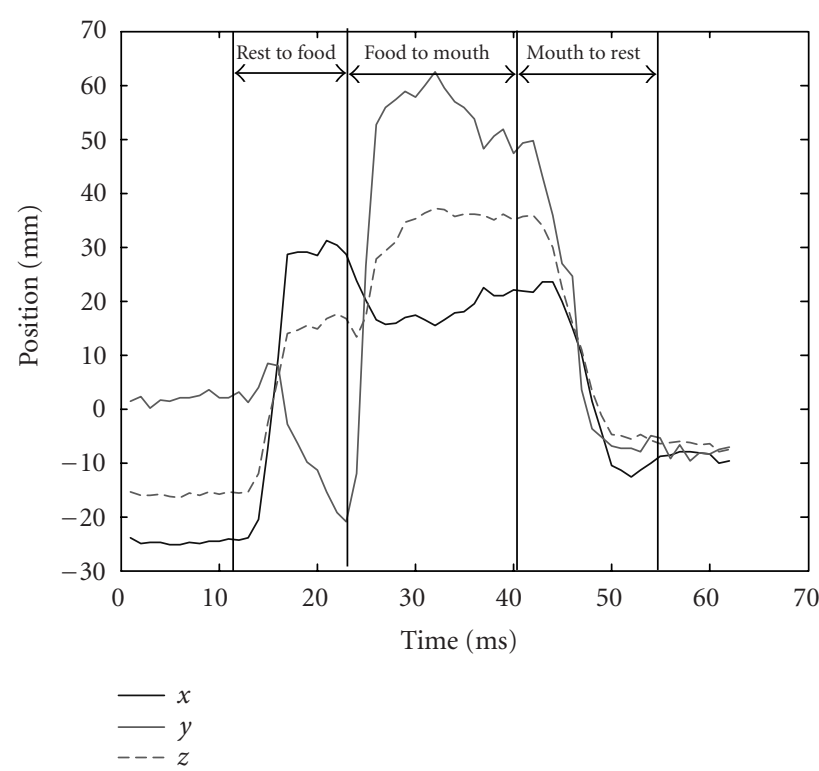

FIGURE 1: Segmentation of the reaching trajectories: reach from rest to food, reach from food to mouth, and reach from mouth to rest positions (taken from [7]).

instability in the NMF algorithm. In addition, to prevent the error criterion from focusing too much on neurons that simply fire frequently (although the temporal structure of their activity might not be significant for the task), the bin counts in each row (i.e., for each neuron) of the data matrix are normalized to have the unit length in its two norms. In general, if $n$ neurons are considered for a total of $m$ time instances, the data matrix $\mathbf{X}$ has dimension $(10 n) \times m$. Since the entries of the data matrix are bin counts, they are guaranteed to be nonnegative. Accounting for 71 or 65 movements, there are $m=2143$ time instances for monkey- 1 and $m=2521$ for monkey-2.

\subsection{Analysis of factorization process}

In the application of NMF to a given neural firing matrix, there are several important issues that must be addressed: the selection of the number of bases, the uniqueness of the NMF solution, and understanding how NMF can find local structures of neural firing activity.

The problem of the choice of the number of bases can be addressed in the framework of model selection. A number of model selection techniques (e.g., the cross-validation) can be utilized for finding the optimal number of bases. In this paper, we choose to adopt a selection criterion that has been recently developed for clustering. The criterion is called the index $I$, which has been used to indicate the cluster 
validity [15]. This index has shown consistent performance of selecting the true number of clusters for various experimental settings. The index $I$ is composed of three factors as

$$
I(r)=\left(\frac{1}{r} \cdot \frac{E_{1}}{E_{r}} \cdot D_{r}\right)^{p}
$$

where $E_{r}$ is the approximation error (Frobenius norm) for $r$ bases, and $D_{r}$ is the maximum Euclidean distance between bases such that

$$
D_{r}=\max _{i, j=1}^{r}\left\|\mathbf{w}_{i}-\mathbf{w}_{j}\right\|
$$

The optimal $r$ is the one that maximizes $I(r)$. We will utilize this index to determine the optimal $r$ for NMF with $p=1$.

Donoho and Stodden have shown that a unique solution of NMF is possible under certain conditions [16]. They have shown through a geometrical interpretation of NMF that if the data are not strictly positive, there can be only one set of nonnegative bases which spans the data in the positive orthant. With an articulated set of images obeying three rules (a generative model, linear independence of generators, and factorial sampling), they showed NMF identifies the generators or "parts" of images. If we consider our neuronal bin-count matrix, each row contains many zero entries (zero bin counts) even after removing nonfiring neurons since most neurons do not fire continuously once in every 100-millisecond window during the entire training set. Therefore, our neuronal data are not strictly positive. This implies that the existence of a unique set of nonnegative bases for the neuronal bin-count matrix is warranted. The question still remains if the NMF basis vectors can find the generative firing patterns for the neural population by meeting the three conditions mentioned above. Here, we discuss the neuronal bin-count data with respect to these conditions.

As stated previously, we have demonstrated through sensitivity analysis that the specific neuronal subsets from the PP, PMd, and M1 regions were sequentially involved in deriving the output of the predictive models during reaching movements [7]. Hence, the bin-count data for the reaching movement will contain increasing firing activity of the specific neuronal subset on local partitions of the trajectory. Due to binning, it is possible that more than one firing pattern is associated with a single data sample. This analysis leads to a generative model for the binned data in which data samples are generated by linear combination of the specific firing patterns with nonnegative coefficients. Also, these firing patterns will be linearly independent since the neuronal subset in each firing patterns tends to modulate firing rates only for the local part of trajectory. The third condition of factorial sampling can be approximately satisfied by the repetition of movements in which the variability of a particular firing pattern is observed during the entire data set. However, a more rigorous analysis is necessary to support the argument that the set of firing patterns is complete in factorial terms. Therefore, we expect that the NMF solutions may be slightly variable reflecting the ambiguity in the completeness of factorial sampling. This might be overcome by collecting more data for reaching movements, and will be pursued in future studies.

\subsection{Case studies}

The NMF algorithm is applied to the described neuronal data matrix prepared using ten taps, $n=91$ neurons for monkey1 (after eliminating the neurons that do not fire through the entire training set) and $n=52$ neurons for monkey2. The NMF algorithm with 100 independent runs results in $r=5$ bases for both monkey- 1 and monkey- 2 datasets for which the index $I$ is maximized. The means and the standard deviations of the normalized cost (Frobenius norm of error between approximation and the given data matrix divided by the Frobenius norm of the data only) for 100 runs are $0.8399 \pm 0.001$ for monkey- 1 data and $0.7348 \pm 0.002$ for monkey-2 data. This implies that the algorithm approximately converges to the same solution with different initial conditions (although not sufficient).

In Figure 2, we show the resulting basis vectors (columns of $\mathbf{W}$ ) for the bin counts (presented in matrix form where columns are different neurons and rows are different delays), as well as their corresponding time-varying encoding coefficients (rows of $\mathbf{H}$ ) superimposed on the reaching trajectory coordinates of three consecutive movements. Based on the assumption that the neuronal bin-count data approximately satisfy the three conditions for the identification of the generators, the NMF basis vectors determine the sequence of spatiotemporal firing patterns representing the firing modulation of the specific neuronal subsets during the course of the reaching movement. Alternatively, we can say that NMF discovers these latent firing patterns of neural population by optimal linear approximation of the data with few bases [9]. For example, from the two basis vectors each corresponding to two primates in the left panel of Figure 2, we observe that firings of the neurons in group- $b$ are followed by firings of the neurons in group- $a$ (the bright activity denoted by $b$ occurs earlier in time than the activity denoted by $a$, since increasing values in the vertical axis of each basis indicates going further back in time). Thus, NMF effectively determines and summarizes this sparse firing pattern that involves a group of neurons firing sequentially. Their relative average activity is also indicated by the relative magnitudes of the entries of this particular basis.

Using these time-synchronized neural activity and hand trajectory recordings, it is also possible to discover relationships between firing patterns and certain aspects of the movement. We can assess the repeatability of a certain firing pattern summarized by a basis vector by observing the time-varying activity of the corresponding encoding signal (the corresponding row of $\mathbf{H}$ ) in time. An increase in this coefficient corresponds to a larger emphasis to that basis in reconstructing the original neural activity data. In the right panel of Figure 2, we observe that all bases are activated regularly in time by their corresponding encoding signals (at different time instances and at different amplitudes). For example, the first basis for monkey- 1 is periodically activated to the same amplitude, whereas the activation amplitude of 


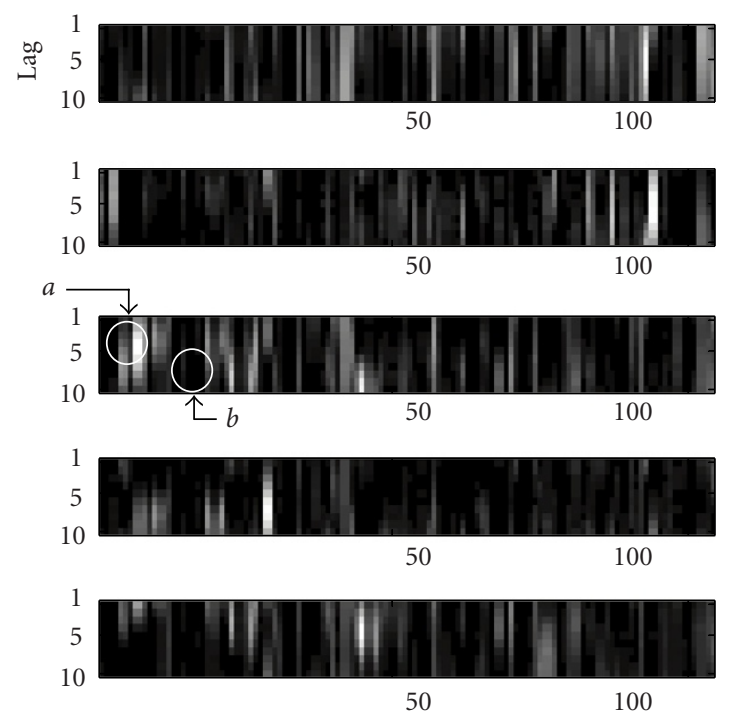

Neuron index

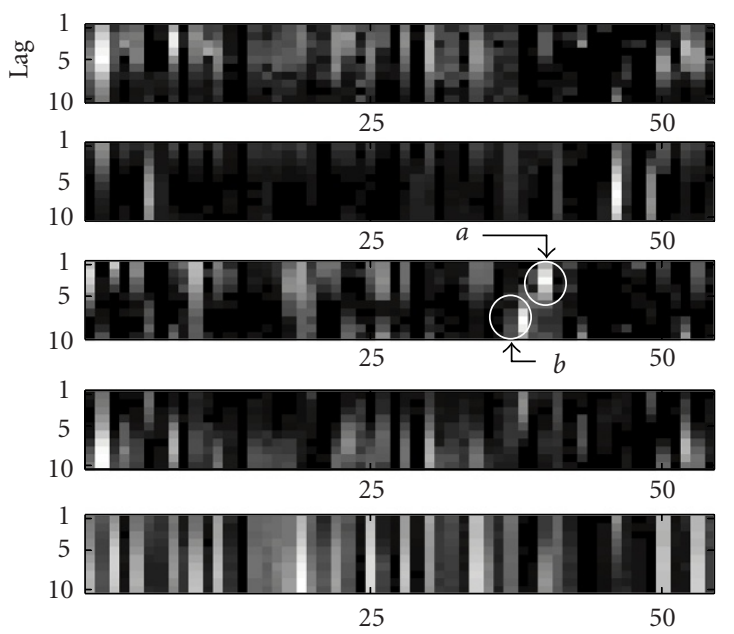

Neuron index

(a)

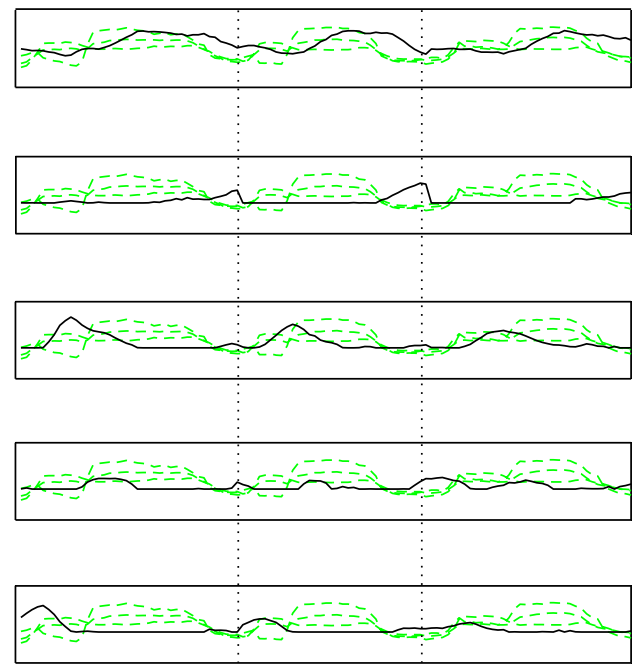

Time
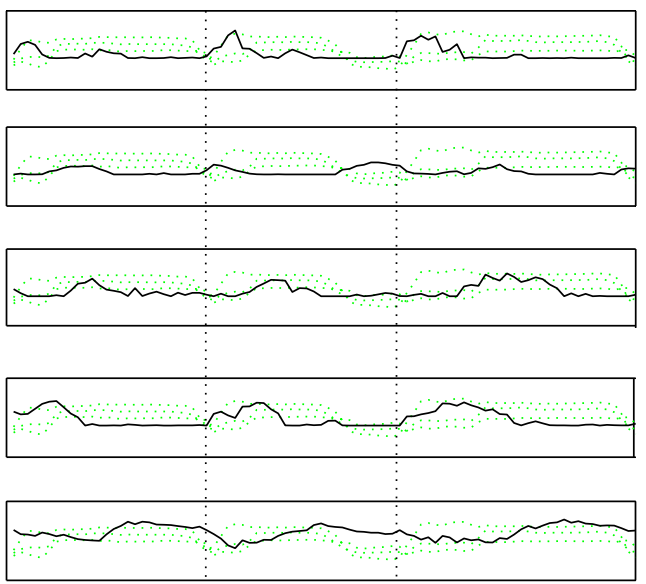

Time

(b)

Figure 2: (a) The five bases for monkey-1 (top) and monkey-2 (bottom). (b) Their corresponding encoding signals (thick solid line) overlaid on the 3-dimensional coordinates of the reaching trajectory (dotted lines) for three consecutive representative reaching tasks (separated by the dashed lines). Note that the encoding signals are scaled to be in the same order of the magnitude of the reaching trajectory for the visual purpose.

the third basis varies in every movement, which might indicate a change in the role of the corresponding neuronal firing pattern in executing that particular movement. The periodic activation of encodings also indicates the bursting nature of the spatiotemporal repetitive patterns. Hence, the NMF bases tend to encode synchronous and bursting spatiotemporal patterns of neural firing activity.

From the NMF decomposition, we observe certain associations between the activities of neurons from different cortical regions and different segments of the reaching trajectory. In particular, an analysis of the monkey- 1 data based on Figure 2 indicates that neurons in PP and M1 (array 1) repeat similar firing patterns during the reach from rest to food. This assessment is based on the observation that bases three, four, and five, which involve firing activities from neurons in these regions, are repeatedly activated by the increased amplitude of their respective encoding coefficients. Similarly, neurons in M1 (array 2) are repeatedly activated during the reach to and from the mouth (bases one and two). These observations are consistent with our previous analyses that were conducted through trained input-output models (such as the Wiener filter and RMLP) [7]. Table 2 compares the neurons, which were observed to have the highest sensitivity from trained models, and the neurons that have the largest magnitudes in each NMF basis. This comparison is based on monkey-1 dataset. We can see that neurons from NMF are a subset of neurons obtained from the sensitivity analysis. It is also worth stating that NMF basis provides more information than the model-based sensitivity analysis since it determines the synchronous spatiotemporal patterns while 
TABLE 2: Comparison of important neurons (examined in the monkey-1 dataset).

\begin{tabular}{ccccc}
\hline Regions & PP & M1(area 1) & PMd & M1(area 2) \\
\hline The high sensitive neurons through RMLP & $4,5,7,22,26,29$ & 38,45 & None & 93,94 \\
The largest-magnitude neurons in NMF bases & 7,29 & 45 & None & 93,94 \\
\hline
\end{tabular}

TABle 3: Performance evaluation of the Wiener filter and the mixture of multiple models based on NMF.

\begin{tabular}{|c|c|c|c|c|c|c|}
\hline & $\mathrm{CC}(\mathrm{x})$ & $\mathrm{CC}(\mathrm{y})$ & $\mathrm{CC}(\mathrm{z})$ & $\operatorname{MSE}(\mathrm{x})$ & $\operatorname{MSE}(\mathrm{y})$ & $\operatorname{MSE}(\mathrm{z})$ \\
\hline \multicolumn{7}{|c|}{ Monkey-1 } \\
\hline Wiener filter & 0.5772 & 0.6712 & 0.7574 & 0.4855 & 0.3468 & 0.2460 \\
\hline NMF mixture & 0.7147 & 0.7078 & 0.8076 & 0.2711 & 0.2786 & 0.1627 \\
\hline \multicolumn{7}{|c|}{ Monkey-2 } \\
\hline Wiener filter & 0.3737 & 0.4304 & 0.6192 & 0.3050 & 0.7405 & 0.2882 \\
\hline NMF mixture & 0.4974 & 0.5041 & 0.6916 & 0.2354 & 0.5400 & 0.2112 \\
\hline
\end{tabular}

the sensitivity analysis only determines individual important neurons. Finally, we would like to reiterate that the analysis presented here is solely based on the data, which means that this analysis does not need to train a specific model to investigate the neural population organization.

\subsection{Modeling improvement for BMI using NMF}

We will demonstrate a simple example showing the improved BMIs performance in predicting hand positions by utilizing NMF. We will compare the performance of two systems; the Wiener filter directly applied to the original spike count data and the mixture of multiple linear filters based on the NMF bases and encodings.

The straight Wiener filter is directly applied to the neural firing data to estimate the three coordinates of the primate's hand position. The Wiener filter has been a standard model for BMIs, and many other approaches have been compared with it [19]. With nine delays, the input dimensionality of the filter is 910 for monkey-1 or 510 for monkey-2 (discarding inactive (no firing) neural channels). Then we add a bias to each input vector to estimate the $y$-intercept. The weights of the filter are estimated by the Wiener-Hopf equation as

$$
\mathbf{W}=\mathbf{R}^{-1} \mathbf{P},
$$

where $\mathbf{R}$ is a $911 \times 911$ (or $511 \times 511$ for monkey-2) input correlation matrix, and $\mathbf{P}$ is a $911 \times 3$ (or $511 \times 3$ for monkey2) input-output cross-correlation matrix.

The mixture of multiple models employs the NMF encodings as mixing coefficients. An NMF basis is used as a window function for the corresponding local model. Therefore, each model sees a given input vector through a different window and uses the windowed input vector to produce the output. Then the NMF encodings are used to combine each model's output to produce the final estimate of the desired hand position vector. This can be described in the following equation:

$$
\hat{d}_{c}(n)=\sum_{k=1}^{K} h_{k}(n)\left(\mathbf{z}_{k}(n)^{T} \mathbf{g}_{k, c}+b_{k, c}\right),
$$

where $h_{k}(n)$ is an NMF encoding coefficient for the $k$ th basis at $n$th column (i.e., time index), $\mathbf{g}_{k, c}$ is the weight vector of the $k$ th model for the $c$ th coordinate $(c \in[x, y, z])$, and $b_{k, c}$ is the $y$-intercept of the $k$ th model for the $c$ th coordinate. $\mathbf{z}_{k}(n)$ is the input vector windowed by the $k$ th NMF basis. Its $i$ th element is given by

$$
z_{k, i}(n)=x_{i}(n) \cdot w_{k, i}
$$

Here, $x_{i}(n)$ is the normalized firing count of the neuron $i$ at time instance $n$, and $w_{k, i}$ is the $i$ th element of the $k$ th NMF basis. $\mathbf{g}_{k, c}$ and $b_{k, c}$ can be estimated based on the MSE criterion by using of the stochastic gradient algorithm such as the normalized least mean square (NLMS). The weight update rule of the NLMS for each model is then given by

$$
\begin{aligned}
& \mathbf{g}_{k, c}(n+1)=\mathbf{g}_{k, c}(n)+\frac{\eta}{\beta+\left\|\mathbf{z}_{k}(n)\right\|^{2}} h_{k}(n) e_{c}(n) \mathbf{z}_{k}(n), \\
& b_{k, c}(n+1)=b_{k, c}(n)+\frac{\eta}{\beta+\left\|\mathbf{z}_{k}(n)\right\|^{2}} h_{k}(n) e_{c}(n),
\end{aligned}
$$

where $\eta$ is the learning rate and $\beta$ is the normalization factor. $e_{c}(n)$ is the error between the $c$ th coordinate of the desired response and the model output.

In the experiment, we divided the data samples into 1771 training samples and 372 test samples for monkey-1 dataset and 1739 and 782, respectively, for monkey-2 dataset. The parameters are set as $\{\eta, \beta, K\}=\{0.01,1,5\}$. The entire training data set is presented 60 times sufficient enough for the weights to converge. The performance of the model is evaluated on the test set by two measures; the correlation coefficient (CC) between desired hand trajectory and the model output trajectory, and the mean squared error (MSE) normalized by the variance of the desired response. Table 3 presents the evaluation of the performance of two systems for both monkey-1 and monkey-2 datasets. It shows a significant improvement in generalization performance with the mixture of models based on NMF factorization.

Note that the general performance of models for the monkey-2 dataset is worse than that for the monkey-1 
dataset. The reasons may come from many experimental variables. One of them may be the number of electrodes and the corresponding cortical areas, as we can see in Table 1 that only 32 electrodes were implanted in two areas for monkey-2, while 64 electrodes in four areas for monkey-1.

To quantify the performance difference between the Wiener filter and the mixture of multiple models, we can apply a statistical test based on the mean squared error (MSE) performance metric [17]. By modeling the performance difference in terms of the MSE using short-time windows as a normal random variable, one can apply the t-test to quantify significance. This t-test was applied to both modeling outputs for monkey- 1 and monkey- 2 with $\alpha=0.01$ or $\alpha=0.05$. For both datasets, the null hypothesis was rejected with both significance levels, resulting in the $p$-values of 0.0023 for monkey- 1 and 0.0007 for monkey-2, respectively. Therefore, the statistical test of the performance difference demonstrates that the mixture of multiple models based on NMF improves the performance significantly compared to the standard Wiener filter.

\subsection{Discussions}

The results presented in the previous case study are a representative example of a broader set of NMF experiments performed on this recording. Selection of the number of taps and the number of bases $(r)$ is dependent on the particular stimulus or behavior associated with the neural data. Although we have used a model selection method originally developed for clustering, and did not provide full justification that this index is suitable to NMF, the main motivation is to demonstrate that the problem of selecting the number of bases can be addressed in the context of model selection. This will be pursued in future research.

The number of patterns that can be distinctly represented by NMF is limited by the number of bases. A very small number of bases will lead to the combination of multiple patterns into a single nonsparse basis vector. At the other extreme, a very large number of bases will result in the splitting of a pattern into two or more bases, which have similar encoding coefficient signals in time. In these situations, the bases under consideration can be combined into one basis.

It is intriguing that the mixture of models based on NMF generalizes better than the Wiener filter despite the fact that the mixture contains much more model parameters. However, each model in the mixture receives the inputs processed by the sparse basis vector. Therefore, each model learns the mapping between only a particular subset of neurons and hand trajectories, and the effective number of parameters for each model is much less than the total number of input variables. Moreover, further overfitting is avoided by combining the outputs of local models by the sparse encodings of NMF.

\section{CONCLUSIONS}

Nonnegative matrix factorization is a novel and relatively new tool for analyzing the data structure when nonnegativity constraints are imposed. In BMIs the neural inputs are processed by grouping the firings into bin counts. Since the bin counts are always positive, we hypothesized that NMF would be appropriate for analyzing the neural activity. The experimental results and the analysis presented in this paper showed that we could find repeated patterns in neuronal activity that occurred in synchrony with the reaching behavior and was automatically and efficiently represented in a set of sparse bases. The sparseness of the bases indicates that only a small number of neurons exhibit repeated firing patterns that are influential in reconstructing the original neural activity matrix.

As presented in [10], NMF provides local bases of the objects, while principal component analysis (PCA) provides global bases. In our preliminary experiments of PCA for the same data, we have observed that PCA only found the most frequently firing neurons, which may not be related to the behavior. Therefore, NMF can find local representation of the neural firing data, and this property of NMF can be more effective than PCA for BMIs where firing activities of different cortical areas are collected.

Lee and Seung have claimed in their paper that the statistical independence among the encodings of independent component analysis (ICA) forces the basis to be holistic [10]. And, if local parts of the neural activity occur together at the same time, the complicated dependencies between the encodings would not be captured by the ICA algorithm. However, we have observed that the NMF encodings seem to be uncorrelated over the entire movement. Hence, ICA with some nonnegative constraints (e. g., nonnegative ICA [18], the ICA model with nonnegative basis [19], and nonnegative sparse coding [20]) may yield interesting encodings of the neural firing activities. Further studies will present the comparison between NMF and these constrained ICA algorithms applied for BMIs.

While NMF is found to be a useful tool for analyzing neural data to find repeatable activity patterns, there are still several issues when using NMF for neural data analysis. Firstly, the method only detects patterns of activity, but it is known that the inactivity of a neuron could often indicate response to a stimulus or cause a behavior. An analysis based on NMF will fail to identify such neurons. Next, the nontationary characteristics of neural activities would make it difficult for NMF to find fixed spatiotemporal firing patterns. Since the neural ensemble function tends to change over neuronal space and time such that different spatio-temporal firing patterns may be involved for the same behavioral output, we may have to continuously adapt NMF factors to track those changes. This motivates us to consider a recursive algorithm of NMF, which will enable us to adapt NMF factors online. It will be covered in the future study.

In our application of NMF, we demonstrated that the NMF learning algorithm resulted in similar Frobenious norm of the error matrix for 100 runs obtained with different initial conditions. However, this does not necessarily mean that the resulted factors are similar with small variance. Therefore, we need to quantify the similarity of the NMF results with different initializations. An alternative is to employ other methods to obtain the global solution such as genetic or simulated annealing algorithms. This will be presented in a follow-up report. 


\section{ACKNOWLEDGMENTS}

The authors would like to thank Johan Wessberg for collecting the data used in this paper. This work was supported by the DARPA project no. N66001-02-C-8022.

\section{REFERENCES}

[1] J. Wessberg, C. R. Stambaugh, J. D. Kralik, et al., "Real-time prediction of hand trajectory by ensembles of cortical neurons in primates," Nature, vol. 408, no. 6810, pp. 361-365, 2000.

[2] D. W. Moran and A. B. Schwartz, "Motor cortical activity during drawing movements: population representation during spiral tracing," Journal of Neurophysiology, vol. 82, no. 5, pp. 2693-2704, 1999.

[3] J. K. Chapin, K. A. Moxon, R. S. Markowitz, and M. A. L. Nicolelis, "Real-time control of a robot arm using simultaneously recorded neurons in the motor cortex," Nature Neuroscience, vol. 2, no. 7, pp. 664-670, 1999.

[4] M. D. Serruya, N. G. Hatsopoulos, L. Paninski, M. R. Fellows, and J. P. Donoghue, "Brain-machine interface: instant neural control of a movement signal," Nature, vol. 416, no. 6877, pp. 141-142, 2002.

[5] J. C. Sanchez, S.-P. Kim, D. Erdogmus, et al., "Input-output mapping performance of linear and nonlinear models for estimating hand trajectories from cortical neuronal firing patterns," in Proc. 12th IEEE International Workshop on Neural Networks for Signal Processing, pp. 139-148, Martigny, Switzerland, September 2002.

[6] S. Darmanjian, S.-P. Kim, M. C. Nechyba, et al., "Bimodal brain-machine interface for motor control of robotic prosthetic," in Proc. IEEE/RSJ International Conference on Intelligent Robots and Systems (IROS '03), vol. 4, pp. 3612-3617, Las Vegas, Nev, USA, October 2003.

[7] J. C. Sanchez, D. Erdogmus, Y. N. Rao, et al., "Interpreting neural activity through linear and nonlinear models for brain machine interfaces," in Proc. 25th Annual International Conference of the IEEE Engineering in Medicine and Biology Society, vol. 3, pp. 2160-2163, Cancun, Mexico, September 2003.

[8] J. M. Carmena, M. A. Lebedev, R. E. Crist, et al., "Learning to control a brain-machine interface for reaching and grasping by primates," PLoS Biology, vol. 1, no. 2, pp. 1-16, 2003.

[9] D. D. Lee and H. S. Seung, "Algorithms for non-negative matrix factorization," in Advances in Neural Information Processing Systems 13, T. K. Leen, T. G. Dietterich, and V. Tresp, Eds., pp. 556-562, MIT Press, Cambridge, Mass, USA, 2001.

[10] D. D. Lee and H. S. Seung, "Learning the parts of objects by non-negative matrix factorization," Nature, vol. 401, no. 6755, pp. 788-791, 1999.

[11] D. Guillamet, M. Bressan, and J. Vitrià, "A weighted nonnegative matrix factorization for local representations," in Proc. IEEE Computer Society Conference on Computer Vision and Pattern Recognition (CVPR '01), vol. 1, pp. 942-947, Kauai, Hawaii, USA, December 2001.

[12] A. d'Avella and M. C. Tresch, "Modularity in the motor system: decomposition of muscle patterns as combinations of time-varying synergies," in Advances in Neural Information Processing Systems 14, T. G. Dietterich, S. Becker, and Z. Ghahramani, Eds., pp. 629-632, MIT Press, Cambridge, Mass, USA, 2002.

[13] J. C. Sanchez, From cortical neural spike trains to behavior: modeling and analysis, Ph.D. dissertation, Department of Biomedical Engineering, University of Florida, Gainesville, Fla, USA, 2004.
[14] M. A. L. Nicolelis, A. A. Ghazanfar, B. M. Faggin, S. Votaw, and L. M. Oliveira, "Reconstructing the engram: simultaneous, multisite, many single neuron recordings," Neuron, vol. 18, no. 4, pp. 529-537, 1997.

[15] U. Maulik and S. Bandyopadhyay, "Performance evaluation of some clustering algorithms and validity indices," IEEE Trans. Pattern Anal. Machine Intell., vol. 24, no. 12, pp. 1650-1654, 2002.

[16] D. Donoho and V. Stodden, "When does non-negative matrix factorization give a correct decomposition into parts?" in Advances in Neural Information Processing Systems 16, S. Thrun, L. K. Saul, and B. Schölkopf, Eds., pp. 1141-1148, MIT Press, Cambridge, Mass, USA, 2004.

[17] S.-P. Kim, J. C. Sanchez, Y. N. Rao, et al., "A Comparison of optimal MIMO linear and nonlinear models for brain-machine interfaces," submitted to Neural Computation, 2004.

[18] M. Plumbley, "Conditions for nonnegative independent component analysis," IEEE Signal Processing Lett., vol. 9, no. 6, pp. 177-180, 2002.

[19] L. Parra, C. Spence, P. Sajda, A. Ziehe, and K.-R. Müller, "Unmixing hyperspectral data," in Advances in Neural Information Processing Systems 12, S. A. Solla, T. K. Leen, and K.-R. Müller, Eds., pp. 942-948, MIT Press, Cambridge, Mass, USA, 2000.

[20] P. O. Hoyer, "Non-negative sparse coding," in Proc. 12th IEEE International Workshop on Neural Networks for Signal Processing, pp. 557-565, Martigny, Switzerland, September 2002.

Sung-Phil Kim was born in Seoul, South Korea. He received a B.S. degree from the Department of Nuclear Engineering, Seoul National University, Seoul, South Korea, in 1994. In 1998, he entered the Department of Electrical and Computer Engineering, University of Florida, in pursuit of Master of Science degree. He joined the Computational NeuroEngineering Laboratory as a Research Assistant in 2000. He also re-

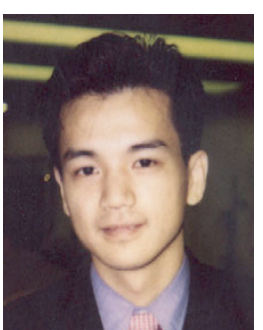
ceived an M.S. degree in December 2000 from the Department of Electrical and Computer Engineering, University of Florida. From 2001, he continued to pursue a Ph.D. degree in the Department of Electrical and Computer Engineering, University of Florida under supervision of Dr. Jose C. Principe. In the Computational NeuroEngineering Laboratory, he has investigated the decoding models and the analytical methods for brain-machine interfaces.

Yadunandana N. Rao was born in Mysore, India. He received his B.E. degree in electronics and communication engineering from the University of Mysore, India, in August 1997, and the M.S. and Ph.D. degrees in electrical and computer engineering from the University of Florida, Gainesville, Fla, in 2000 and 2004, respectively. From May 2000 to January 2001, he worked as a Design Engineer at GE Medical Systems, Wis. Currently he is a Senior Engineer at Motorola, Fla. His research interests include adaptive signal processing theory, algorithms and analysis, neural networks for signal processing, and biomedical applications. 
Deniz Erdogmus received his B.S. degrees in electrical engineering and mathematics in 1997, and his M.S. degree in electrical engineering, with emphasis on systems and control, in 1999, all from the Middle East Technical University, Turkey. He received his $\mathrm{Ph} . \mathrm{D}$. degree in electrical engineering from the University of Florida, Gainesville, in 2002. Since 1999, he has been with the Computational NeuroEngineering Labora-

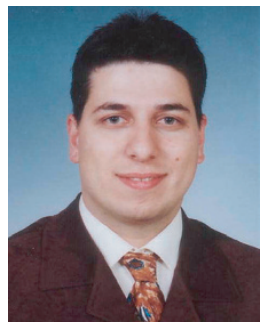
tory, University of Florida, working with Jose Principe. His current research interests include information-theoretic aspects of adaptive signal processing and machine learning, as well as their applications to problems in communications, biomedical signal processing, and controls. He is the recipient of the IEEE SPS 2003 Young Author Award, and is a Member of IEEE, Tau Beta Pi, and Eta Kappa Nu.

Justin C. Sanchez received a B.S. degree with highest honors in engineering science along with a minor in biomechanics from the University of Florida in 2000. From 1998 to 2000, he spent three years as a Research Assistant in the Department of Anesthesiology, University of Florida. In 2000, he joined the Department of Biomedical Engineering and Computational NeuroEngineering Laboratory, the University of

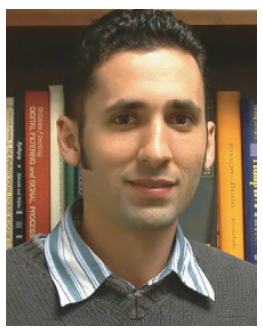
Florida. In the spring of 2004, he completed both his M.E. and Ph.D. degrees in biomedical signal processing working on the development of modeling and analysis tools for brain-machine interfaces. He is currently a Research Assistant Professor in the Department of Pediatrics, Division of Neurology, the University of Florida. His neural engineering electrophysiology laboratory is currently developing neuroprosthetics for use in the research and clinical settings.

Miguel A. L. Nicolelis was born in Sao Paulo, Brazil, in 1961. He received his M.D. and Ph.D. degrees from the University of Sao Paulo, Brazil, in 1984 and 1988, respectively. After postdoctoral work at Hahnemann University, Philadelphia, he joined Duke University, where he now codirects the Center for Neuroengineering and is a Professor of neurobiology, biomedical engineering, and psychological and brain sci-

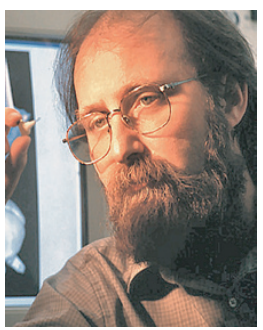
ences. His laboratory is interested in understanding the general computational principles underlying the dynamic interactions between populations of cortical and subcortical neurons involved in motor control and tactile perception.

Jose C. Principe is a Distinguished Professor of electrical and computer engineering and biomedical engineering at the University of Florida where he teaches advanced signal processing, machine learning, and artificial neural networks (ANNs) modeling. $\mathrm{He}$ is a BellSouth Professor and the Founder and Director of the University of Florida Computational NeuroEngineering Laboratory (CNEL). His primary area of interest

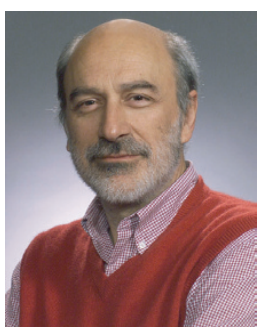
is processing of time-varying signals with adaptive neural models. The CNEL has been studying signal and pattern recognition principles based on information-theoretic criteria (entropy and mutual information). He is an IEEE Fellow. He is a Member of the ADCOM of the IEEE Signal Processing Society, a Member of the Board of Governors of the International Neural Network Society, and the Editor-in-Chief of the IEEE Transactions on Biomedical Engineering. He is a Member of the Advisory Board of the University of Florida Brain Institute. He has more than 90 publications in refereed journals, 10 book chapters, and 200 conference papers. He directed 35 Ph.D. dissertations and 45 Master theses. He recently wrote an interactive electronic book entitled Neural and Adaptive Systems: Fundamentals Through Simulation published by John Wiley and Sons. 